\title{
Recovery of rough surface in ducting medium from grazing angle scattered wave
}

Y. Chen, O. Rath Spivack, and M. Spivack

Citation: Journal of Applied Physics 124, 084901 (2018); doi: 10.1063/1.5044605

View online: https://doi.org/10.1063/1.5044605

View Table of Contents: http://aip.scitation.org/toc/jap/124/8

Published by the American Institute of Physics

\section{Articles you may be interested in}

Electron emission by long and short wavelength lasers: Essentials for the design of plasmonic photocathodes Journal of Applied Physics 124, 083101 (2018); 10.1063/1.5031882

Instability of crystal/melt interface in Si-rich SiGe Journal of Applied Physics 124, 085104 (2018); 10.1063/1.5038755

Focusing light through random scattering media by simulated annealing algorithm Journal of Applied Physics 124, 083104 (2018); 10.1063/1.5019238

Effects of surface roughness on surface charge accumulation characteristics and surface flashover performance of alumina-filled epoxy resin spacers

Journal of Applied Physics 124, 083302 (2018); 10.1063/1.5043239

Two-dimensional electron density visualization over plasma flare in vacuum breakdown process

Journal of Applied Physics 124, 083301 (2018); 10.1063/1.5035100

Localization of nonlinear spin waves in magnetic multilayers

Journal of Applied Physics 124, 085301 (2018); 10.1063/1.5037211

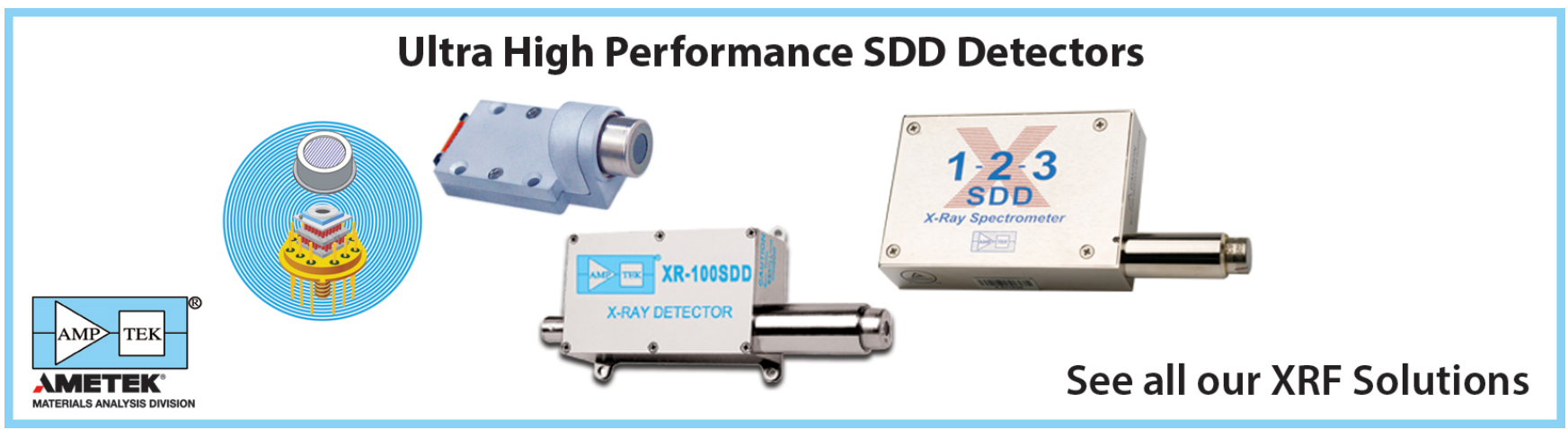




\title{
Recovery of rough surface in ducting medium from grazing angle scattered wave
}

\author{
Y. Chen, ${ }^{1, a)}$ O. Rath Spivack, ${ }^{2, b)}$ and M. Spivack ${ }^{2, c)}$ \\ ${ }^{1}$ Department of Engineering, University of Cambridge, Cambridge CB2 1PZ, United Kingdom \\ ${ }^{2}$ Department of Applied Mathematics and Theoretical Physics, University of Cambridge, Cambridge CB3 OWA, \\ United Kingdom
}

(Received 14 June 2018; accepted 10 August 2018; published online 29 August 2018)

\begin{abstract}
A method is developed for rough surface reconstruction using fields scattered at grazing angles in a medium with a linearly varying refractive index and Neumann boundary condition. This regime represents a ducting medium, bounded by a perfectly conducting surface with a TM incident field or an acoustically hard surface. This significantly extends the iterated marching method, based upon the parabolic integral equation for forward-scattered field components [Chen and Spivack, J. Opt. Soc. Am. A 35, 504-513 (2018)]. The approach, which uses a fixed frequency, is able to accurately recover multiscale surfaces and is found to be robust with respect to measurement noise and localized perturbations. Published by AIP Publishing. https://doi.org/10.1063/1.5044605
\end{abstract}

\section{INTRODUCTION}

Wave scattering by rough surfaces plays a key role in a wide range of applications ${ }^{1-5}$ and has been studied extensively. The recovery of surface topography and environmental parameters from scattered data remains an important and challenging area. ${ }^{6,7}$ A variety of mathematical and physically based approaches have been employed, notably smallparameter approximations, ${ }^{8,9}$ iterative methods, ${ }^{10-12}$ integral equations, ${ }^{13-16}$ time domain point source, ${ }^{17}$ and multiview. ${ }^{18}$ In sea state and surface profile retrieval, Doppler and backscattered multiple frequency radar measurements have also been used. $^{19-22}$

Often, however, the medium possesses a refractive index profile which greatly complicates the wave scattering, as is the case in the above-ocean evaporation duct ${ }^{23,24}$ for radar applications and the SOFAR channel or other underice profiles affecting sound propagation in the ocean. ${ }^{25,26}$ This variation may produce channelling which exacerbates multiple scattering, and very little progress has been made on the surface inverse problem in such situations.

For general incident angles, the scattered field obeys the Helmholtz boundary integral equation. ${ }^{1,3,27}$ However, when most energy is forward-scattered as it is at near-grazing angles, wave propagation is well described by the parabolic equation. ${ }^{28}$ Applying this to the governing Green's function allows the Helmholtz integral equations to be replaced by the parabolic integral equation method, ${ }^{29,30}$ which is the key to the method developed here.

In this paper, an algorithm is developed for reconstruction of a rough surface $h(x)$ in a two-dimensional medium with a linearly depth-dependent refractive index, exploiting the properties of grazing angle scatter. A Neumann boundary condition is assumed. This approach extends recent work on

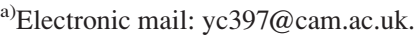

b)Electronic mail: or100@cam.ac.uk.

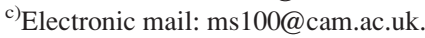

the Dirichlet case for a constant medium. ${ }^{31}$ Here, we make use of the Neumann form of the parabolic equation Green's function for a varying medium derived by Uscinski, ${ }^{32,33}$ which has received little attention in the literature.

To derive the inversion algorithm the problem is formulated as an integral equation in the unknown surface field $\Phi$ considered as a function of the surface. This is coupled to an expression relating $h$ to $\Phi$, and the system is solved directly by numerical inversion. The Volterra form of the integral equation allows us to find the surface progressively along the propagation direction and to substitute the values back into the kernel. The incident wave field and the linear profile are assumed known, together with scattered data along a line parallel to the mean surface level. With this information, the scattering integral may be treated as an integral equation and can be solved accordingly.

Scattered data are obtained by two independent methods: The first is simply via the above parabolic integral equations including the Green's function of the varying medium. The second is by exploiting the powerful image medium method, first proposed by Tappert ${ }^{34}$ for the parabolic equation regime. This is a differential equation formulation, in which the rough surface adjacent to the half-space is replaced by a combined real and image medium, separated by a "virtual" boundary in which the image medium has an artificial varying refractive index which depends on the surface, and, crucially, can take into account arbitrary refractive index variations in the real medium.

In Sec. II, the parabolic integral equation method for scattering by a surface with Neumann boundary condition and linear profile is reviewed. The two methods for generating the scattered field are summarised in Sec. III. The equations for the inverse problem are formulated in Sec. IV, and the algorithm for solution of these equations is derived. Results of numerical experiments are given in Sec. V, where we study a range of multiscale rough surfaces and initial conditions and examine the influence of measurement noise on the results. 


\section{MATHEMATICAL FORMULATION AND GREEN'S FUNCTION}

In this section, we give the parabolic equation Green's function for the medium with a linear depth-dependent refractive index profile $e^{32,33}$ and review the boundary integral equations upon which the inversion algorithm is based. The generation of scattered field measurements, both by direct treatment of these equations and by the image method, will be described in Sec. III.

Consider a two-dimensional time-harmonic scalar wave $p$, resulting from scattering by a rough surface of a field incident at low grazing angle. We assume the Neumann boundary condition, i.e., vanishing of the normal derivative, corresponding to an acoustically hard surface, or TM polarized electromagnetic field and perfect conductivity. The coordinate axes are $x$ and $z$, where $x$ is the horizontal $x \geq 0$ and $z$ is the vertical, directed into the medium (see Fig. 1).

The mean surface level is taken to be at $z=0$. The source is centred about $\mathbf{r}=\left(0, z_{0}\right)$, with wave number $k$. The rough surface itself is denoted $h(x)$, so that $h$ has mean zero. In the numerical examples, $h$ is drawn from an ensemble of normally distributed and statistically stationary processes, with rms (root means square) surface height denoted by $\Sigma$. (This statistical description is used for convenience and is not central to the algorithm.)

Since the wave field propagates predominantly in one direction, it has a slowly varying part (or reduced wave) $\psi$ defined by

$$
\psi(x, z)=p(x, z) \exp (-i k x) .
$$

Incident and scattered components $\psi_{i}$ and $\psi_{s}$ such that $\psi$ $=\psi_{i}+\psi_{s}$ are defined analogously. The area of surface illumination is assumed to be negligible for negative $x$, so that $\psi_{i}(x, h(x))=0$ for $x \leq 0$, corresponding, for example, to a Gaussian beam.

Under the assumption of small angles of incidence and scattering, the parabolic form of the Green's function can be introduced (see Thorsos ${ }^{29}$ )

$$
G\left(x, z ; x^{\prime}, z^{\prime}\right)=\frac{1}{2}\left[\frac{i}{2 \pi k\left(x-x^{\prime}\right)}\right]^{1 / 2} \exp \left[\frac{i k\left(z-z^{\prime}\right)^{2}}{2\left(x-x^{\prime}\right)}\right],
$$

when $x^{\prime}<x$ and $G=0$ otherwise. This Green's function is derived under the assumption of forward scattering, i.e., that the field obeys the parabolic wave equation

$$
\psi_{x}-\frac{i}{2 k} \psi_{z z}=0,
$$

which holds under the given small-angle assumptions.

$$
\text { linear profile }
$$$$
\mathrm{n}=\mathrm{n}_{0}(1+\mathrm{az})
$$

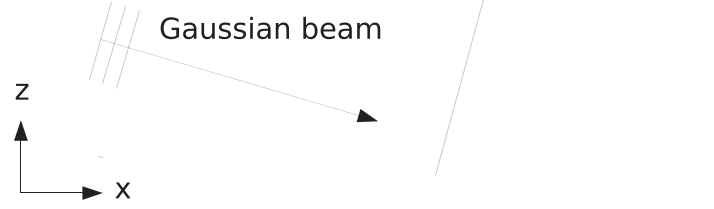

FIG. 1. Schematic view of the scattering configuration.
Governing integral equations for the parabolic equation method can be derived for general boundary conditions ${ }^{29}$ and specialised to Dirichlet ${ }^{29,30}$ and Neumann. ${ }^{32,33}$ For the Neumann case treated here, these become

$$
\psi_{i}(\mathbf{r})=\frac{\phi(\mathbf{r})}{2}+\int_{0}^{x} \frac{\partial G\left(\mathbf{r} ; \mathbf{r}^{\prime}\right)}{\partial z^{\prime}} \phi\left(\mathbf{r}^{\prime}\right) d x^{\prime},
$$

where $\phi(x)=\psi(x, h(x))$ is the total field on the surface, both $\mathbf{r}=(x, h(x)), \mathbf{r}^{\prime}=\left(x^{\prime}, h\left(x^{\prime}\right)\right)$ lie on the surface, and

$$
\psi_{s}(\mathbf{r})=-\int_{0}^{x} \frac{\partial G\left(\mathbf{r} ; \mathbf{r}^{\prime}\right)}{\partial z^{\prime}} \phi\left(\mathbf{r}^{\prime}\right) d x^{\prime},
$$

where $\mathbf{r}^{\prime}$ is again on the surface and $\mathbf{r}$ is now an arbitrary point in the medium. Here, the normal derivative of the Green's function has been replaced by a vertical derivative under the same approximations.

It is the one-way nature of the Green's function which gives rise to the finite upper limit of integration in Eqs. (3) and (4). The accuracy has been examined by Thorsos ${ }^{29}$ and subsequent authors. These equations are not applicable to situations in which backscattering is significant. In the above equations, $\phi$ is defined only at surface points, so that it may be considered here as a function just of $x$. The incident field is taken here to be a Gaussian beam of initial width $w$, centred at a distance $z_{0}$ from the surface. For simplicity, the beam will be assumed to be directed parallel to the surface

$$
\psi_{i}(x, z),=\frac{i}{2 k} \frac{w}{\left(w^{2}+2 i x / k\right)^{1 / 2}} \exp \left[-\frac{\left(z-z_{0}\right)^{2}}{w^{2}+2 i x / k}\right] .
$$

This field impinges upon the surface as it propagates; the pattern of illumination along a flat surface rises from zero to a peak and decays with $1 / \sqrt{x}$. The assumption of zero grazing angle is not crucial; similar results hold for incidence at small nonzero angles.

A parabolic (one-way) form $G_{p}$ of the Green's function can be derived ${ }^{32}$ for a linear profile, in which the exponent becomes modified. Suppose that refractive index is a function of vertical coordinate $n(z)=n_{0}(1+a z)$, where $n_{0}$ is the constant reference value and $a$ is the "strength" of the profile. Then, we can write

$$
G_{p}=G \times G_{2},
$$

where

$$
G_{2}\left(\mathbf{r} ; \mathbf{r}^{\prime}\right)=\exp \left[\frac{i k}{2}\left(a\left(z+z^{\prime}\right)\left(x-x^{\prime}\right)-\frac{a^{2}\left(x-x^{\prime}\right)^{3}}{12}\right)\right] .
$$

The Gaussian incident field subsequently changes due to the profile term. The Gaussian beam can be obtained by the linear profile Green's function and integrating over all source points, which is

$$
\begin{aligned}
\psi_{i}(x, z)= & \frac{i}{2 k} \frac{w}{\left(w^{2}+2 i x / k\right)^{1 / 2}} \exp \left[-i k\left(a x z+\frac{a^{2} x^{3}}{6}\right)\right] \\
& \times \exp \left[-\frac{\left(z-z_{0}+a x^{2} / 2\right)^{2}}{w^{2}+2 i x / k}\right] .
\end{aligned}
$$




\section{DIRECT PROBLEM AND GENERATION OF SCATTERED FIELD}

Scattered field data are generated by two independent approaches, both valid in the parabolic equation regime. The first is via the parabolic integral equations above, and the second by the image medium method of Tappert Nghiem$\mathrm{Phu},{ }^{34}$ in which the rough surface is replaced by an extended medium and scattering is solved by a partial differential equation. The latter provides an independent means of producing scattered field data, while the integral equations serve to motivate the inversion algorithm.

In what follows, both the direct and the inverse scattering problems are considered on a domain of finite extent. The rough surface may be considered as a segment of an extended (infinite or semi-infinite) surface which may (but need not) be otherwise flat. Any discontinuities or edges to the left of $x=0$ do not influence the data as they are not insonified/illuminated. Edges to the right, however, may cause significant backscattering which will violate the parabolic equations assumptions used throughout.

\section{A. Treatment of integral equations}

The parabolic integral equation approach has been welldocumented elsewhere, ${ }^{29,30,33}$ and we only need to give a brief overview here. For convenience, we can express the pair of Eqs. (3) and (4) in operator notation as, say,

$$
\begin{aligned}
\psi_{i} & =A \phi, \\
\psi_{s} & =B \phi,
\end{aligned}
$$

where $A, B$ represent the operators on the right-hand-sides of Eqs. (3) and (4), respectively. From integral equation (9), we obtain the surface field as $\phi=A^{-1} \psi_{i}$ and can then substitute this into the integral (10) to obtain the field $\psi_{s}(\mathbf{r})$ at any point $\mathbf{r}$ in the medium. By discretizing the surface into (say) $n$ evenly spaced $x$-coordinates, these operators become $n \times n$ matrices. The inversion of the integral equation is then equivalent to the inversion of such a matrix.

Once $\phi$ has been found on the surface, the field $\phi$ in the medium is calculated straightforwardly by substitution into integral (10).

\section{B. Image medium method}

In contrast to the integral equation approach, the image medium method ${ }^{34-37}$ does not require the Green's function for the medium, which may have refractive index variations. Instead, the real (physical) half-space is extended to include an image space, and a transformation is applied to the governing differential equation giving rise to a virtual refractive index. This refractive index is the physical one $n(z)$ in the real medium but has an additional component depending on $n(z)$ and the surface function $h(x)$ in the image space. In addition, if the source is located at $z=f(0)+z_{0}$, then this is augmented by an image source located at $z=f(0)-z_{0}$. It will be easy to see that for a flat surface this reduces to the standard image method for wave propagation in a medium with a profile.
The field is assumed to obey the parabolic wave equation, above a rough surface. The rough surface and lower half-space are then replaced by an image medium, with an image source, whose refractive index profile reflects the properties of the surface. After some algebraic manipulations, the following equation is derived:

$$
\frac{\partial \psi}{\partial x}=\frac{i}{2 \kappa_{0}} \frac{\partial^{2} \psi}{\partial z^{2}}+\frac{i \kappa_{0}}{2} N(x, z) \psi,
$$

where

$$
\psi(x, z)= \begin{cases}\psi_{R}(x, z) & z>h(x) \\ \psi_{I}(x, z) e^{2 i \kappa_{0} h^{\prime}(x)[z-h(x)]} & z<h(x)\end{cases}
$$

and

$$
N(x, z)=\left\{\begin{array}{cc}
n^{2}(x, z)-1 & z>h(x) \\
n^{2}(x,-z+2 h(x))-1 & \\
+4 h^{\prime \prime}(x)[z-h(x)] & z<h(x) .
\end{array}\right.
$$

The functions $\psi_{R}, \psi_{I}$ are the "real" and "image" solutions, and as in Ref. 36 it can be shown that this system obeys the required boundary conditions. This differential equation is then solved by a straightforward marching technique.

The procedure for the horizontally polarised incident field is identical. However, since the modified image medium method solves in a sense a more general problem, we shall present the results of the vertical polarisation case. (The results are qualitatively similar, except that the rough surface causes greater loss of energy into diffuse directions for vertical polarization, and the interference patterns, most evident for flat surfaces, are shifted.) This will be done in Sec. V.

\section{INVERSE PROBLEM: ALGORITHM AND THEORETICAL TREATMENT}

Suppose that the scattered field is known along an interval $[0, L]$ at some distance $z$ from the surface. Throughout the numerical experiments below, we will use the same interval for both measured data and the domain of reconstruction as discussed further in Sec. V. Under the forward-scattering approximation, the scattered field at each $x$ is assumed to depend only on surface points $x^{\prime} \leq x$. We can thus attempt to recover the surface $h(x)$ over the full interval.

The inversion approach at each step is in two stages. The first is to calculate the total wave (employing at the initial step an ad hoc guess). The second reconstructs the surface by "marching" in range. In the successive improvement, this is repeated a small number of times and is found to be remarkably well-suited to this regime.

We define $H\left(\mathbf{r} ; \mathbf{r}^{\prime}\right)=\frac{\partial G\left(\mathbf{r} ; \mathbf{r}^{\prime}\right)}{\partial z^{\prime}}$ with

$$
H\left(\mathbf{r} ; \mathbf{r}^{\prime}\right)=\alpha \frac{z-z^{\prime}}{\left(x-x^{\prime}\right)^{3 / 2}} \exp \left[\frac{i k\left(z-z^{\prime}\right)^{2}}{2\left(x-x^{\prime}\right)}\right],
$$

where $\alpha=-(i / 2) \sqrt{i k / 2 \pi}$ corresponding to uniform medium problem and 


$$
\begin{aligned}
H_{p}\left(\mathbf{r} ; \mathbf{r}^{\prime}\right)= & \alpha\left(H_{1}-H_{2}\right), \\
H_{1}\left(\mathbf{r} ; \mathbf{r}^{\prime}\right)= & \frac{z-z^{\prime}}{\left(x-x^{\prime}\right)^{3 / 2}} \exp \left[\frac { i k } { 2 } \left(\frac{\left(z-z^{\prime}\right)^{2}}{x-x^{\prime}}\right.\right. \\
& \left.\left.+a\left(z+z^{\prime}\right)\left(x-x^{\prime}\right)-\frac{a^{2}\left(x-x^{\prime}\right)^{3}}{12}\right)\right] \\
H_{2}\left(\mathbf{r} ; \mathbf{r}^{\prime}\right)= & \frac{a}{2}\left(x-x^{\prime}\right)^{1 / 2} \exp \left[\frac { i k } { 2 } \left(\frac{\left(z-z^{\prime}\right)^{2}}{x-x^{\prime}}\right.\right. \\
& \left.\left.+a\left(z+z^{\prime}\right)\left(x-x^{\prime}\right)-\frac{a^{2}\left(x-x^{\prime}\right)^{3}}{12}\right)\right],
\end{aligned}
$$

corresponding to the linear profile problem. The integration domain $[0, L]$ is discretized by $\mathrm{N}$ nodes with $x_{r}$ for $r=0,1, \ldots, N$, where $x_{0}=0$ and $x_{N}=L$. The space between each node is denoted as $\delta$.

\section{A. Recovery of surface wavefield}

With an initial guess, calculating the surface wavefield is like the direct problem. Once the kernel $G$ is known, Eq. (3) can be regarded as a Volterra integral equation in $\phi$. After discretization, Eq. (3) can be written as a sum of $n$ subintegrals at every node $x_{n} \in\left[x_{1}, x_{N}\right]$ on the integral domain

$$
\begin{aligned}
\psi_{i}\left(\mathbf{r}_{\mathbf{n}}\right) & =\frac{\phi}{2}\left(\mathbf{r}_{\mathbf{n}}\right)+\sum_{r=1}^{n} \int_{x_{r-1}}^{x_{r}} H\left(\mathbf{r}_{\mathbf{n}} ; \mathbf{r}^{\prime}\right) \phi\left(x^{\prime}\right) d x^{\prime} \\
& =\sum_{r=1}^{n} \int_{x_{r-1}}^{x_{r}}\left(H\left(\mathbf{r}_{\mathbf{n}} ; \mathbf{r}^{\prime}\right)+\frac{\delta\left(x_{n} ; x^{\prime}\right)}{2}\right) \phi\left(x^{\prime}\right) d x^{\prime}
\end{aligned}
$$

where $\mathbf{r}_{\mathbf{n}}=\left(x_{n}, h\left(x_{n}\right)\right)$ and $\mathbf{r}^{\prime}=\left(x^{\prime}, h\left(x^{\prime}\right)\right)$. If we assume $\phi$ varies slowly over each subinterval compared to Green's function, then $\phi$ can be treated as constant and taken out of the subintegrals

$$
\psi_{i}\left(\mathbf{r}_{\mathbf{n}}\right) \approx \sum_{r=1}^{n} \phi\left(X_{r}\right) \int_{x_{r-1}}^{x_{r}}\left(H\left(\mathbf{r}_{\mathbf{n}} ; \mathbf{r}^{\prime}\right)+\frac{\delta\left(x_{n} ; x^{\prime}\right)}{2}\right) d x^{\prime},
$$

where $X_{r}=\left(x_{r-1}+x_{r}\right) / 2$. For $n=1,2, \ldots, N, N$ linear equations are obtained from Eq. (14), which results in a $N \times N$ linear system. Denote two vectors of size $N, \Psi_{i} \in \mathbb{C}^{N}$ and $\Phi \in \mathbb{C}^{N}$ with

$$
\begin{aligned}
\Psi_{i} & =\left[\psi_{i}\left(x_{1}, h\left(x_{1}\right)\right), \psi_{i}\left(x_{2}, h\left(x_{2}\right)\right), \ldots, \psi_{i}\left(x_{N}, h\left(x_{N}\right)\right)\right], \\
\Phi & =\left[\phi\left(X_{1}\right), \phi\left(X_{2}\right), \ldots, \phi\left(X_{N}\right)\right] .
\end{aligned}
$$

Hence, they are related by

$$
A \Phi=\Psi_{i},
$$

where

$$
A(n, r)= \begin{cases}\int_{x_{r-1}}^{x_{r}} H\left(x_{n}, h\left(x_{n}\right) ; x^{\prime}, h\left(x^{\prime}\right)\right) d x^{\prime}, & r<n \\ \int_{x_{n-1}}^{x_{n}} H\left(x_{n}, h\left(x_{n}\right) ; x^{\prime}, h\left(x^{\prime}\right)\right) d x^{\prime}+\frac{1}{2}, & r=n\end{cases}
$$

for $1 \leq r \leq n \leq N$. $A$ is a lower triangular matrix, whose inversion is computationally efficient.

\section{Uniform medium}

First for $r<n$, there is no singularity in the integral. Under the assumption that the exponential term varies slowly and can be treated as constant, the integral becomes

$$
\alpha E\left(h\left(x_{n}\right) ; n, r\right) \int_{x_{r-1}}^{x_{r}} \frac{h\left(x_{n}\right)-h\left(x^{\prime}\right)}{\left(x_{n}-x^{\prime}\right)^{3 / 2}} d x^{\prime},
$$

where

$$
E(z ; n, r)=\exp \left[\frac{i k\left(z-h\left(X_{r}\right)\right)^{2}}{2\left(x_{n}-X_{r}\right)}\right] .
$$

Apply the Taylor expansion on $h$ with $h\left(x^{\prime}\right)=h\left(x_{r-1}\right)$ $+h^{\prime}\left(x_{r-1}\right)\left(x^{\prime}-x_{r-1}\right)$, then the remaining integral has the form

$$
\begin{aligned}
\int_{x_{r-1}}^{x_{r}} & \frac{h\left(x_{n}\right)-h\left(x^{\prime}\right)}{\left(x_{n}-x^{\prime}\right)^{3 / 2}} d x^{\prime} \\
= & \int_{x_{r-1}}^{x_{r}} \frac{h\left(x_{n}\right)-h\left(x_{r-1}\right)-h^{\prime}\left(x_{r-1}\right)\left(x^{\prime}-x_{r-1}\right)}{\left(x_{n}-x^{\prime}\right)^{3 / 2}} \\
= & \left(h\left(x_{n}\right)-h\left(x_{r-1}\right)\right) \int_{x_{r-1}}^{x_{r}} \frac{d x^{\prime}}{\left(x_{n}-x^{\prime}\right)^{3 / 2}} \\
& -h^{\prime}\left(x_{r-1}\right) \int_{x_{r-1}}^{x_{r}} \frac{x^{\prime}-x_{n}+x_{n}-x_{r-1}}{\left(x_{n}-x^{\prime}\right)^{3 / 2}} d x^{\prime} \\
= & L\left(h\left(x_{n}\right) ; n, r\right) \int_{x_{r-1}}^{x_{r}} \frac{d x^{\prime}}{\left(x_{n}-x^{\prime}\right)^{3 / 2}} \\
& +h^{\prime}\left(x_{r-1}\right) \int_{x_{r-1}}^{x_{r}} \frac{d x^{\prime}}{\left(x_{n}-x^{\prime}\right)^{1 / 2}},
\end{aligned}
$$

where

$$
L(z ; n, r)=z-h\left(x_{r-1}\right)-h^{\prime}\left(x_{r-1}\right)\left(x_{n}-x_{r-1}\right) .
$$

For $r=n$, there is a singularity in the integral. Apply the same Taylor expansion with $h\left(x^{\prime}\right)=h\left(x_{n}\right)+h^{\prime}\left(x_{n}\right)\left(x^{\prime}-x_{n}\right)$, then the integral becomes

$$
\int_{x_{n-1}}^{x_{n}} \alpha \frac{h^{\prime}\left(x_{n}\right)}{\left(x_{n}-x^{\prime}\right)^{1 / 2}} \exp \left[\frac{i k}{2} h^{\prime}\left(x_{n}\right)^{2}\left(x_{n}-x^{\prime}\right)\right] d x^{\prime} .
$$

To eliminate the singularity, change of variable by

$$
\xi=\left(x_{n}-x^{\prime}\right)^{1 / 2},
$$

then $d x^{\prime}=-2 \xi d \xi$. Equation (17) turns to

$$
\begin{gathered}
\int_{\sqrt{\delta}}^{0} \alpha \frac{h^{\prime}\left(x_{n}\right)}{\xi} \exp \left[\frac{i k}{2} h^{\prime}\left(x_{n}\right)^{2} \xi^{2}\right](-2 \xi) d \xi \\
=\int_{0}^{\sqrt{\delta}} 2 \alpha h^{\prime}\left(x_{n}\right) \exp \left[\frac{i k}{2} h^{\prime}\left(x_{n}\right)^{2} \xi^{2}\right] d \xi .
\end{gathered}
$$

Apply the formula

$$
\int e^{\mu x^{2}} d x=-\frac{i}{2} \frac{\sqrt{\pi}}{\sqrt{\mu}} \operatorname{erf}(i x \sqrt{\mu})
$$


where erf is the error function. With $\mu=\frac{i k}{2} h^{\prime}\left(x_{n}\right)^{2}$, then the integral becomes

$$
-\alpha h^{\prime}\left(x_{n}\right) \frac{\sqrt{\pi}}{\sqrt{\mu}} i \operatorname{erf}(\sqrt{\mu} \sqrt{\delta} i) .
$$

Therefore, we can approximate the matrix $A$ by

$$
A(n, r)=\left\{\begin{array}{rlr}
\alpha E & \left(h\left(x_{n}\right) ; n, r\right) & \\
\quad \times\left[L\left(h\left(x_{n}\right) ; n, r\right) \int_{x_{r-1}}^{x_{r}} \frac{d x^{\prime}}{\left(x_{n}-x^{\prime}\right)^{3 / 2}}\right. & \\
\left.+h^{\prime}\left(x_{r-1}\right) \int_{x_{r-1}}^{x_{r}} \frac{d x^{\prime}}{\left(x_{n}-x^{\prime}\right)^{1 / 2}}\right], & r<n \\
-\alpha h^{\prime}\left(x_{n}\right) \frac{\sqrt{\pi}}{\sqrt{\mu}} i \operatorname{erf}(\sqrt{\mu} \sqrt{\delta} i), & r=n .
\end{array}\right.
$$

\section{Medium with linear profile}

The treatment with the linearly varying profile in the medium is similar. The integral containing $H_{1}$ is dealt with similar to the previous case. For $r<n$, the same assumption is applied here that the exponential parts vary slowly compared to other terms and can be taken out of each integral. Denote it as

$$
\begin{aligned}
& F(z ;, n, r) \\
& \quad=\exp \left[\frac{i k}{2}\left(a\left(z+h\left(X_{r}\right)\right)\left(x_{n}-X_{r}\right)-\frac{a^{2}\left(x_{n}-X_{r}\right)^{3}}{12}\right)\right] .
\end{aligned}
$$

Together with the approximation (16), the integral of $H_{1}$ becomes

$$
\begin{aligned}
& E\left(h\left(x_{n}\right) ; n, r\right) F\left(h\left(x_{n}\right) ; n, r\right) \\
& \quad \times\left[L\left(h\left(x_{n}\right) ; n, r\right) \int_{x_{r-1}}^{x_{r}} \frac{d x^{\prime}}{\left(x_{n}-x^{\prime}\right)^{3 / 2}}\right. \\
& \left.\quad+h^{\prime}\left(x_{r-1}\right) \int_{x_{r-1}}^{x_{r}} \frac{d x^{\prime}}{\left(x_{n}-x^{\prime}\right)^{1 / 2}}\right] .
\end{aligned}
$$

The singularity also exists when $r=n$; the same singularity arguments are employed. First, apply the Taylor expansion on $h$, then substitute Eq. (18); the integral becomes

$$
\int_{x_{n-1}}^{x_{n}} H_{1} d x^{\prime}=-h^{\prime}\left(x_{n}\right) \frac{\sqrt{\pi}}{\sqrt{\mu}} i[\operatorname{erf}(\sqrt{\mu} \sqrt{\delta} i)] F\left(h\left(x_{n}\right) ; n, n\right) .
$$

On the other hand, the $H_{2}$ integral can be evaluated immediately via taking out the exponential parts, for $r<n$, the integral becomes

$$
\frac{a}{2} E\left(h\left(x_{n}\right) ; n,, r\right) F\left(h\left(x_{n}\right) ; n, r\right) \int_{x_{r-1}}^{x_{r}}\left(x_{n}-x^{\prime}\right)^{1 / 2} d x^{\prime} .
$$

And the singularity part for $r=n$ is followed by the same way

$$
\int_{x_{n-1}}^{x_{n}} H_{2} d x^{\prime}=F\left(h\left(x_{n}\right) ; n, n\right) \int_{0}^{\sqrt{\delta}} a \xi^{2} \exp \left[\frac{i k}{2}\left(h^{\prime}\left(x_{n}\right)^{2} \xi^{2}\right)\right] d \xi .
$$

By further changing variables setting $\zeta=\xi^{2}$, this becomes

$$
\frac{a}{2} F\left(h\left(x_{n}\right) ; n, n\right) \int_{0}^{\delta} \sqrt{\zeta} \exp \left[\frac{i k}{2} h^{\prime}\left(x_{n}\right)^{2} \zeta\right] d \zeta .
$$

Apply the equation

$$
\int \sqrt{x} e^{\mu x} d x=\frac{1}{\mu} \sqrt{x} e^{\mu x}+\frac{i \sqrt{\pi}}{2 \mu^{3 / 2}} \operatorname{erf}(i \sqrt{\mu x})
$$

with the same $\mu=\frac{i k}{2} h^{\prime}\left(x_{n}\right)^{2}$, the integral turns to

$$
\frac{a}{2} F\left(h\left(x_{n}\right) ; n, n\right)\left[\frac{1}{\mu} \sqrt{\delta} e^{\mu \delta}+\frac{i \sqrt{\pi}}{2 \mu^{3 / 2}} \operatorname{erf}(i \sqrt{\mu \delta})\right] .
$$

Finally, the matrix for the linear profile problem is obtained

$$
A(n, r)=\left\{\begin{aligned}
& \alpha E\left(h\left(x_{n}\right) ; n, r\right) F\left(h\left(x_{n}\right) ; n, r\right)\left[L\left(h\left(x_{n}\right) ; n, r\right)\right. \\
& \times \int_{x_{r-1}}^{x_{r}} \frac{d x^{\prime}}{\left(x_{n}-x^{\prime}\right)^{3 / 2}}+h^{\prime}\left(x_{r-1}\right) \int_{x_{r-1}}^{x_{r}} \frac{d x^{\prime}}{\left(x_{n}-x^{\prime}\right)^{1 / 2}} \\
&-\left.\frac{a}{2} \int_{x_{r-1}}^{x_{r}}\left(x_{n}-x^{\prime}\right)^{1 / 2} d x^{\prime}\right], \\
&-\alpha F\left(h\left(x_{n}\right) ; n, n\right)\left[h^{\prime}\left(x_{n}\right) \frac{\sqrt{\pi}}{\sqrt{\mu}} i \operatorname{erf}(\sqrt{\mu} \sqrt{\delta} i)\right. \\
&\left.-\frac{a}{2}\left[\frac{1}{\mu} \sqrt{\delta} e^{\mu \delta}+\frac{i \sqrt{\pi}}{2 \mu^{3 / 2}} \operatorname{erf}(i \sqrt{\mu \delta})\right]\right], \quad r=n .
\end{aligned}\right.
$$

\section{B. Surface reconstruction}

For all $x_{n} \in\left[X_{1}, X_{N}\right]$, the scattered data $\psi_{s}\left(x_{n}, z\right)$ are known at certain height $z$. Expand Eq. (4) by the similar way under the same assumption that $\phi$ can be treated as constant on each subinterval

$$
\psi_{s}\left(x_{n}, z\right)=-\sum_{l=1}^{n} \phi\left(X_{l}\right) \int_{x_{l-1}}^{x_{l}} H\left(x_{n}, z ; x^{\prime}, h\left(x^{\prime}\right)\right) d x^{\prime},
$$

where $X_{l}=\frac{1}{2}\left(x_{l-1}+x_{l}\right)$. Extract the $n-1$ terms on the right to the left, we have

$$
\begin{gathered}
\psi_{s}\left(x_{n}, z\right)+\sum_{l=1}^{n-1} \phi\left(X_{l}\right) \int_{x_{l-1}}^{x_{l}} H\left(x_{n}, z ; x^{\prime}, h\left(x^{\prime}\right)\right) d x^{\prime} \\
=-\phi\left(X_{n}\right) \int_{x_{n-1}}^{x_{n}} H\left(x_{n}, z ; x^{\prime}, h\left(x^{\prime}\right)\right) d x^{\prime} .
\end{gathered}
$$

Let us denote the left hand side as $S_{n}$. If $h\left(X_{l}\right)$ is obtained for $l=1,2, \ldots, n-1$, then $S_{n}$ can be calculated directly as in the previous part, thus $h\left(X_{n}\right)$ can be obtained by solving Eq. (22), which leads to a marching method.

\section{Uniform medium}

First the sum $S_{n}$ can be evaluated via taking the exponential term out together with Eq. (16) 


$$
\begin{aligned}
S_{n}= & \psi_{s}\left(x_{n}, z\right)+\sum_{l=1}^{n} \alpha \phi\left(X_{l}\right) E(z ; n, l)[L(z ; n, l) \\
& \left.\times \int_{x_{l-1}}^{x_{l}} \frac{d x^{\prime}}{\left(x_{n}-x^{\prime}\right)^{3 / 2}}+h^{\prime}\left(x_{l-1}\right) \int_{x_{l-1}}^{x_{l}} \frac{d x^{\prime}}{\left(x_{n}-x^{\prime}\right)^{1 / 2}}\right],
\end{aligned}
$$

Once $S_{n}$ is totally known, Eq. (22) for the uniform medium is

$$
-\phi\left(X_{n}\right) \int_{x_{n-1}}^{x_{n}} \alpha \frac{z-h\left(x^{\prime}\right)}{\left(x_{n}-x^{\prime}\right)^{3 / 2}} \exp \left[\frac{i k}{2} \frac{\left(z-h\left(x^{\prime}\right)\right)^{2}}{x_{n}-x^{\prime}}\right] d x^{\prime}=S_{n} .
$$

Apply a direct approximation on the integral, keep the terms with the unknown $h\left(X_{n}\right)$ to the left, we have

$$
\left(z-h\left(X_{n}\right)\right) E(z ; n, n)=\frac{S_{n}\left(x_{n}-X_{n}\right)^{3 / 2}}{-\alpha \phi\left(X_{n}\right) \delta} .
$$

The problem now transforms into finding the surface from a non-linear equation. A trick can be employed here. If we take modulus both sides, the exponential term disappears immediately; hence, the equation becomes linear with $h\left(X_{n}\right)$

$$
\left|z-h\left(X_{n}\right)\right|=\left|\frac{S_{n}\left(x_{n}-X_{n}\right)^{3 / 2}}{-\alpha \phi\left(X_{n}\right) \delta}\right|
$$

Finally, the surface height can be obtained directly provided the height $z$ is above the whole surface

$$
h\left(X_{n}\right)=z-\left|\frac{S_{n}\left(x_{n}-X_{n}\right)^{3 / 2}}{-\alpha \phi\left(X_{n}\right) \delta}\right| .
$$

\section{Medium with linear profile}

The same treatments can be applied here, and the sum $S_{n}$ is evaluated by

$$
\begin{aligned}
S_{n}= & \psi_{s}\left(x_{n}, z\right)+\sum_{l=1}^{n-1} \alpha \phi\left(X_{l}\right) E(z ; n, l) F(z ; n, l) \\
& \times\left[L(z ; n, l) \int_{x_{l-1}}^{x_{l}} \frac{d x^{\prime}}{\left(x_{n}-x^{\prime}\right)^{3 / 2}}\right. \\
& \left.+h^{\prime}\left(x_{l-1}\right) \int_{x_{l-1}}^{x_{l}} \frac{d x^{\prime}}{\sqrt{x_{n}-x^{\prime}}}-\frac{a}{2} \int_{x_{l-1}}^{x_{l}} \sqrt{x_{n}-x^{\prime}} d x^{\prime}\right] .
\end{aligned}
$$

Equation (22) can be approximated directly by

$$
\begin{aligned}
S_{n}= & -\phi\left(X_{n}\right) \int_{x_{n-1}}^{x_{n}} \alpha\left(H_{1}-H_{2}\right) d x^{\prime} \\
= & -\phi\left(X_{n}\right) \alpha \delta E(z ; n,, n) F(z ; n, n) \\
& \times\left[\frac{z-h\left(X_{n}\right)}{\left(x_{n}-X_{n}\right)^{3 / 2}}-\frac{a}{2}\left(x_{n}-X_{n}\right)^{1 / 2}\right] .
\end{aligned}
$$

Rearrange it and putting $h$ on the left hand side

$$
\left[\frac{z-h\left(X_{n}\right)}{\left(x_{n}-X_{n}\right)^{3 / 2}}-\frac{a}{2} \sqrt{x_{n}-X_{n}}\right] E(z ; n, n) F(z ; n, n)=\frac{S_{n}}{-\alpha \phi\left(X_{n}\right) \delta} .
$$

Taking the modulus both sides, the exponential part can be removed

$$
\left|\frac{S_{n}}{-\alpha \phi\left(X_{n}\right) \delta}\right|=\left|\frac{z-h\left(X_{n}\right)}{\left(x_{n}-X_{n}\right)^{3 / 2}}-\frac{a}{2}\left(x_{n}-X_{n}\right)^{1 / 2}\right| .
$$

We can solve this equation provided $z>h(x)+\frac{a \delta^{2}}{8}$, where $\frac{a \delta^{2}}{8} \ll h(x)$. Finally, the surface height is reconstructed via

$$
h\left(X_{n}\right)=z-\left[\left|\frac{S_{n}}{-\alpha \phi\left(X_{n}\right) \delta}\right|+\frac{a}{2}\left(x_{n}-X_{n}\right)^{1 / 2}\right]\left(x_{n}-X_{n}\right)^{3 / 2} .
$$

\section{Successive improvement}

Since the surface reconstruction can be obtained with some initial guess $h_{0}$, the successive improvement can be employed. Suppose the first surface reconstruction $h_{1}$ is obtained via surface wavefield $\phi_{1}$, it can be substituted back to calculate a new surface wave $\phi_{2}$. Then a new surface $h_{2}$ can be reconstructed with the new surface wave $\phi_{2}$. This process can be repeated. This kind of iterative method is found to work well in improving the performance. In order to get satisfactory surface reconstruction, only a few iterations are needed (typically three). The whole procedure is shown in Algorithm 1.

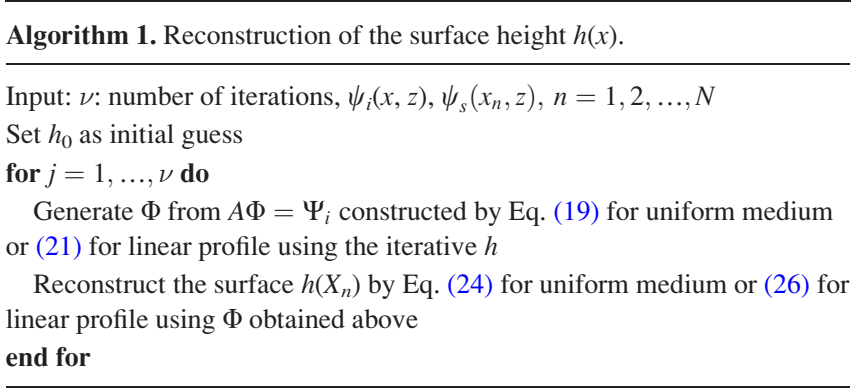

\section{RESULTS}

The reconstruction algorithm has been tested on a range of problems and implemented in Python. The scattered data were obtained by two methods as described in Sec. III, using Fortran for the image method and Python for the integral equation results. In the process of applying the inversions algorithm, the surface functions $h(x)$ are allowed to take complex values for computational convenience; the resulting values are found to have negligibly small imaginary component, which can be discarded. This serves as an additional indirect check on self-consistency.

The random rough surface chosen here is generated computationally from an autocorrelation function (a.c.f.) $\rho(\eta)$ where $\eta=x^{\prime}-x$. The examples shown here use wave number $k=1$ although by renormalising length scales this may represent arbitrary wavelengths. We choose a Gaussiantype a.c.f. 


$$
\rho(\eta)=\sigma^{2} \exp \left(\frac{-\eta^{2}}{l^{2}}\right)
$$

where $l=8 \cong 1.3 \lambda$ is the autocorrelation length and $\rho(0)$ $=\sigma^{2}$ is the variance. A simple way to synthesise the surface is as a superposition of a sinusoidal components with phases chosen uniformly in $[0,2 \pi)$ and applying a filter function to give rise to the chosen autocorrelation function. These surfaces $h(x)$ were generated on a longer segment and then truncated to $x_{i} \in[0, L]$ for evenly spaced elements $x_{i}$ of the computational grid. The peaks exist along the surface at small scales. The typical peak-to-trough of the surface is about 0.5 . The incident Gaussian beam is centred at $z_{0}=22.4$ at zero grazing angle, and initial width is taken to be $w=8$. The maximum range $L$ is taken to be $L=300 \mathrm{~m}$. For this value of $k$, this range is thus $L \cong 50 \lambda$. The initial guess of the inverse problem is chosen as

$$
h_{0}=\sin (0.05 x) / 1000 .
$$

The use of a small non-zero initial function here helps to stabilize the reconstruction. However, it has been found that the results are highly insensitive to the exact form of this initial guess.

The key scattering scales in the direct problem within the parabolic equation regime are the ratio of surface height to autocorrelation length. In the inverse problem, the incident wavelengths can in principle be tuned to optimize the algorithms. On the other hand, the non-dimensional ratio of rms surface height to correlation length is a feature of the particular physical setup which is not under our control.

The source is taken to be a known Gaussian beam, given by Eq. (5). For example at zero grazing in free space, i.e., in the absence of reflecting boundary or refractive index variation, the field will propagate without distortion. Any depthdependent profile will distort the propagating wave. In the case of a reflecting surface $h(x)$ and a linear profile (or indeed any profile increasing with distance from $h(x)$ ), the wave field will be refracted towards the surface, where it will become repeatedly scattered. The contour plot of the modulus of the field is shown in Fig. 2.

\section{A. Reconstruction}

\section{Uniform medium}

The scattered wave field was sampled along a plane at the height $z=0.7$. Computational nodes $x_{i}$ for $i=1, \ldots, N$ were evenly spaced for $N$ ranging between 300 and 800 in different cases. This corresponds to resolution ranging from

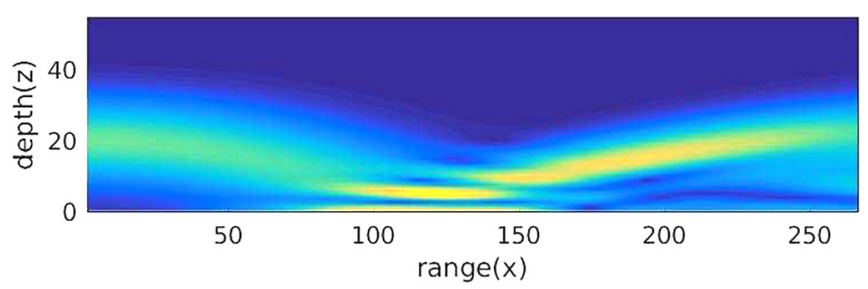

FIG. 2. Plots of the total field amplitude for a Gaussian beam above a flat surface in a medium with the linear profile. around 6 to 16 points per wavelength. The reconstructions $h\left(x_{i}\right)$ are carried out at the same evenly spaced values of $x_{i}$. The use of evenly spaced points at the same horizontal locations for both sampled data and reconstructions is a significant numerical convenience, but is not necessary for the application of the algorithm either theoretically or in practice.

The algorithm was carried out for 3 iterations. The reconstruction at third iteration in each case was found to be clearly satisfactory. It is seen that even though more iterations are applied, the improvement on the surface reconstruction is not so much obvious. Figure 3 shows the reconstructed height plotted against the original surface height at the first and the third iteration.

The approximated surface closely follows the original surface, and most detailed features of the surface are recaptured. At the first iteration, the reconstruction deviates from the original surface mainly at the region of the peaks. This is due to the increasing error caused by the large exponential term. At the third iteration, the error around peaks is significantly reduced since the recovery of the surface wave field improves.

We also test the algorithm with respect to measurement noise, by the addition of white noise to the scattered data. This perturbation ranged from $1 \%$ to $5 \%$ of the rms scattered field amplitude, measured from the sum of squares at the nodes Note that this component was statistically stationary throughout the spatial domain, including regions where the exact data were negligibly small, where it can potentially have a disproportionate effect on the results. Figure 4 shows the reconstruction with 5\% Gaussian noise added. The result contains oscillations throughout the whole domain. However, these oscillations are qualitatively similar to the noise itself. Since in practice the surface is assumed to be smooth on the smallest scale size of the grid, the oscillations shown on the reconstruction can be effectively filtered out. The filtered reconstruction is done by a simple five-point moving average, which is shown in Fig. 4. The filtered surface again convincingly reproduces the exact form. Thus, the marching algorithm exhibits a type of self-regularization, in which large errors at the initial region do not propagate as the reconstruction proceeds along the propagation direction.

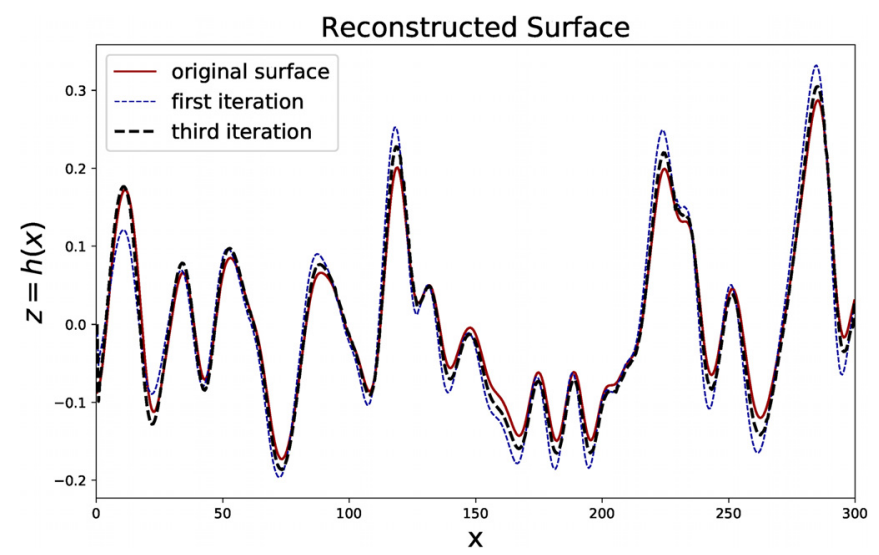

FIG. 3. Plots of rough surface $h$ from inverse problem for the first and third iterations. 

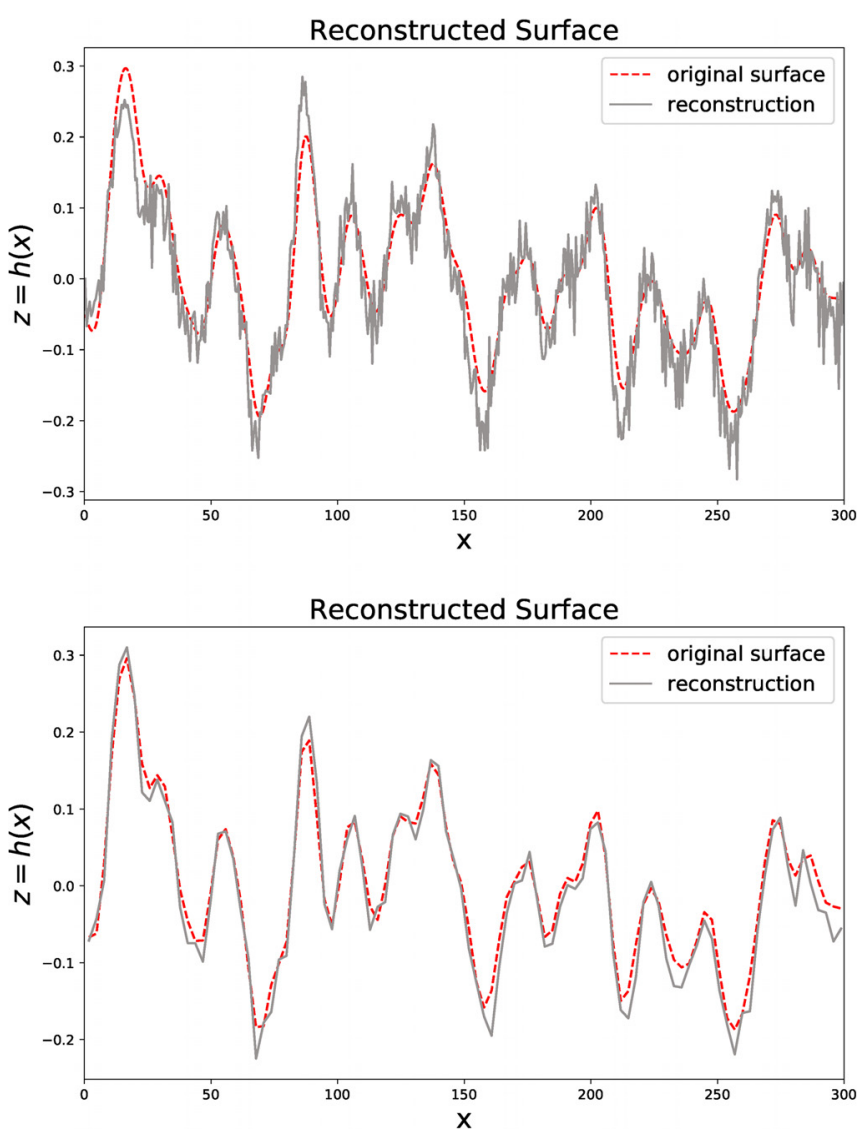

FIG. 4. Comparison of the actual surface reconstruction (upper) with filtered reconstruction (lower) at the third iteration for noise level of 5\% added to scattered data.

\section{Linear profile}

We now consider the case of a medium with non-zero linear profile. The profile parameter is taken as $a=0.015$. The reconstructed surfaces against the original surface at the first and the third iteration are shown in Fig. 5. Apparently, the reconstruction at first iteration is not good enough, especially towards the end of the domain. Some large peaks are present in certain areas. The reason for these large oscillations is that the surface wave field is not good at the area. On the other hand, at the third iteration, the surface settles down

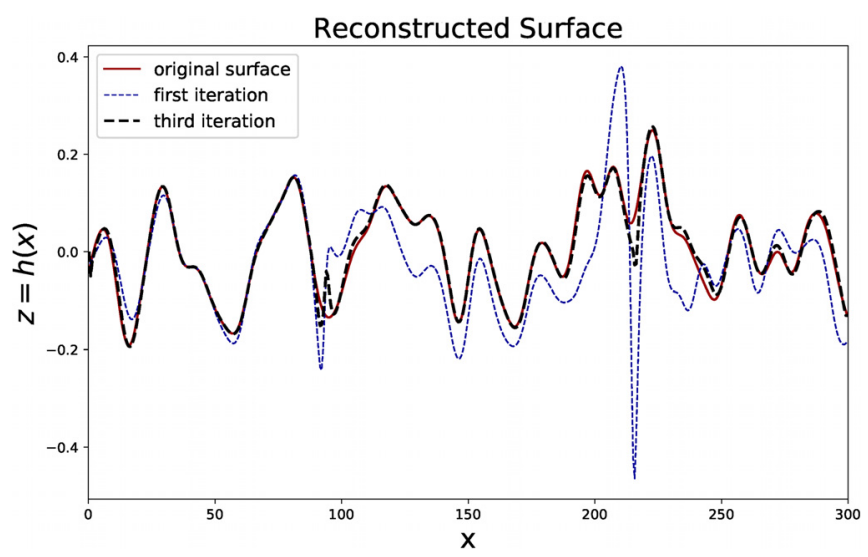

FIG. 5. Plots of rough surface $h$ from inverse problem for the first iteration (dotted) and the third iteration (dashed). and fits the original surface well. The reconstruction tends to stabilize as it progresses to right. It shows that the successive improvement works better in the linear profile case.

For the linear profile problem, we also test the performance with respect to the noise data. Figure 6 gives the reconstruction with $2 \%$ Gaussian noise added and the filtered surface by the same five-point average. Similar results are obtained.

\section{B. Error analysis}

The nature and extent of the errors in the reconstruction surface are immediately evident from the plots. We can also examine the error at each iteration in terms of the $l_{2}$ norm of the residual, given by

$$
e_{j}=\frac{1}{N}\left[\sum_{i=1}^{N}\left(H_{i}-h_{i}^{j}\right)^{2}\right]^{1 / 2},
$$

where $H_{i}$ is the original surface value and $h_{i}^{j}$ is the recovered surface at the $j$ th iteration, measured at the $n$th node. The performance of the algorithm is examined here in terms of three controlling parameters: the number of nodes $N$ (which in turn determines the resolution), the height at which scattered data are measured, and the profile parameter governing the strength of ducting. Table I gives the error with respect to the number of nodes at each iteration. It is clear that the algorithm improves through iteration, and higher resolution
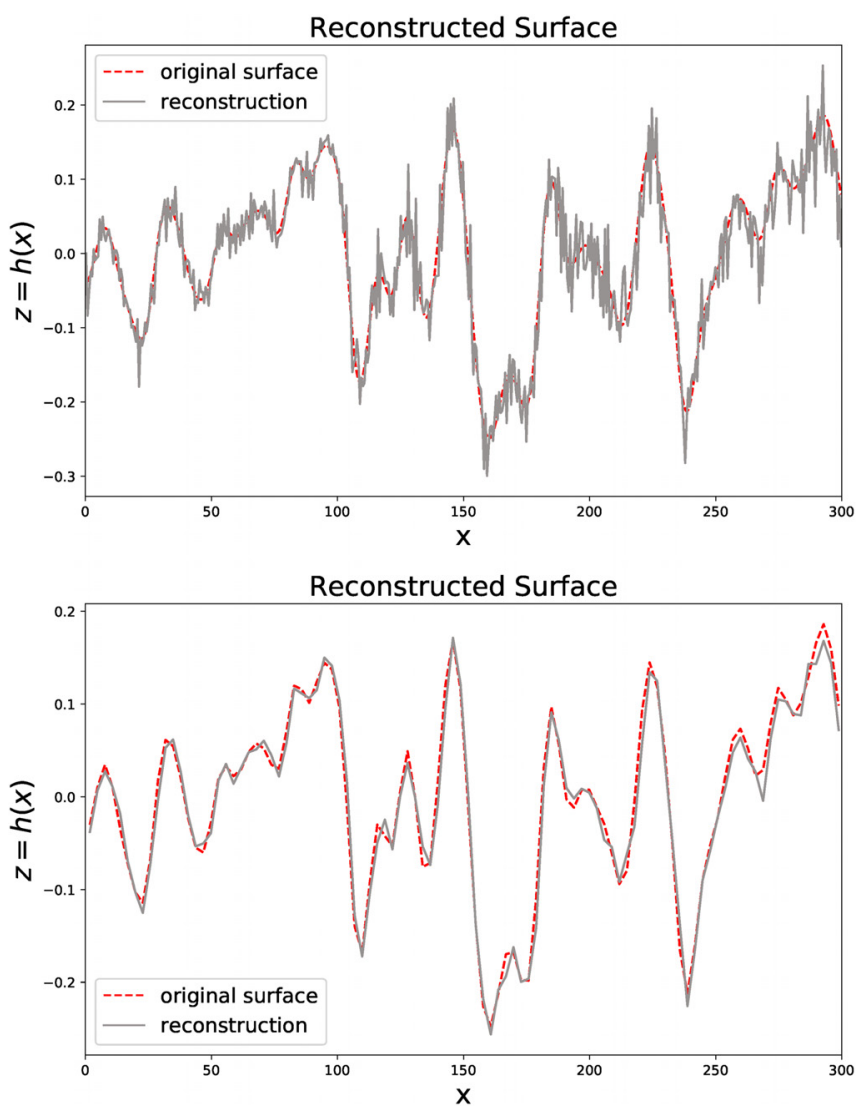

FIG. 6. Comparison of the actual surface reconstruction (upper) with filtered reconstruction (lower) at the third iteration for noise level of $2 \%$ added to scattered data. 
TABLE I. $l_{2}$ error per node for different numbers of nodes.

\begin{tabular}{|c|c|c|c|c|c|c|}
\hline \multirow[b]{2}{*}{ Number of nodes } & \multicolumn{3}{|c|}{ Uniform medium } & \multicolumn{3}{|c|}{ Linear profile } \\
\hline & 300 & 500 & 800 & 300 & 500 & 800 \\
\hline$e_{1}$ & $1.91 \times 10^{-3}$ & $1.35 \times 10^{-3}$ & $7.17 \times 10^{-4}$ & $7.03 \times 10^{-3}$ & $3.28 \times 10^{-3}$ & $2.42 \times 10^{-3}$ \\
\hline$e_{2}$ & $1.25 \times 10^{-3}$ & $6.83 \times 10^{-4}$ & $3.43 \times 10^{-4}$ & $3.17 \times 10^{-3}$ & $1.16 \times 10^{-3}$ & $9.10 \times 10^{-4}$ \\
\hline$e_{3}$ & $1.14 \times 10^{-3}$ & $6.14 \times 10^{-4}$ & $3.24 \times 10^{-4}$ & $2.26 \times 10^{-3}$ & $5.23 \times 10^{-4}$ & $3.36 \times 10^{-4}$ \\
\hline
\end{tabular}

TABLE II. $l_{2}$ error per node for different heights scattered data measured.

\begin{tabular}{|c|c|c|c|c|c|c|}
\hline \multirow[b]{2}{*}{ Height of scattered data } & \multicolumn{3}{|c|}{ Uniform medium } & \multicolumn{3}{|c|}{ Linear profile } \\
\hline & 0.5 & 0.7 & 1.0 & 0.5 & 0.7 & 1.0 \\
\hline$e_{1}$ & $1.30 \times 10^{-3}$ & $1.35 \times 10^{-3}$ & $1.23 \times 10^{-3}$ & $3.12 \times 10^{-3}$ & $3.28 \times 10^{-3}$ & $4.69 \times 10^{-3}$ \\
\hline$e_{2}$ & $8.09 \times 10^{-4}$ & $6.83 \times 10^{-4}$ & $7.55 \times 10^{-4}$ & $9.37 \times 10^{-4}$ & $1.16 \times 10^{-3}$ & $2.29 \times 10^{-3}$ \\
\hline$e_{3}$ & $7.45 \times 10^{-4}$ & $6.14 \times 10^{-4}$ & $7.35 \times 10^{-4}$ & $4.38 \times 10^{-4}$ & $5.23 \times 10^{-3}$ & $1.36 \times 10^{-3}$ \\
\hline
\end{tabular}

TABLE III. $l_{2}$ error per node for different profile parameters.

\begin{tabular}{lcccc}
\hline \hline & \multicolumn{4}{c}{ Linear profile } \\
\cline { 2 - 5 } Profile parameter (a) & 0.03 & 0.02 & 0.015 & 0.010 \\
\hline$e_{1}$ & $7.83 \times 10^{-3}$ & $8.04 \times 10^{-3}$ & $3.28 \times 10^{-3}$ & $2.67 \times 10^{-3}$ \\
$e_{2}$ & $5.06 \times 10^{-3}$ & $3.02 \times 10^{-3}$ & $1.16 \times 10^{-3}$ & $8.43 \times 10^{-4}$ \\
$e_{3}$ & $3.67 \times 10^{-3}$ & $1.23 \times 10^{-3}$ & $5.23 \times 10^{-4}$ & $5.66 \times 10^{-4}$ \\
\hline \hline
\end{tabular}

also gives rise to better reconstruction. The error obtained for different scattered data heights is shown in Table II. In the case of a uniform medium the performance is similar for different scattered data heights. However, for a linear profile, the error increases with height of the measurement plane. Finally Table III presents the error for different values of the profile parameter. As the scattered data height $z$ or the profile parameter $a$ increases, the approximation for the exponential terms $E(z ; n, l)$ and $F(z ; n, l)$ becomes worse, and these is, therefore, a decline in performance. Overall, the results are acceptable giving good agreement, with a relatively small error.

\section{CONCLUSIONS}

We have extended the iterated marching method to the recovery of a rough surface from grazing angle scattered data in a ducting medium with a known linearly varying refractive index. Results are obtained here for surfaces obeying the Neumann boundary condition. To the best of our knowledge, the surface reconstruction problem for a non-uniform medium has not previously been addressed, despite the importance of these regimes in both radar and underwater or under-ice acoustics. The method can in principle be applied to any varying medium provided the Green's function is known, and can be extended to include the Dirichlet boundary (although the Neumann boundary causes greater scattering and has received somewhat less attention in the inverse problems literature). A refractive index profile in the medium can channel the wave to become scattered repeatedly at the surface, thereby increasing the severity of multiple scattering which is already inevitable at grazing angles. Numerical experiments have been conducted for a variety of cases and extremely good agreement has been found between reconstructed and exact surface shapes. We have also examined the effect on the algorithm of significantly perturbing the measured data with white noise, and it is found to be robust. In this case, the initial surface reconstruction follows the correct surface profile plus a noisy component with statistical characteristics similar to the measurement noise which can, therefore, be easily filtered out.

We note that in contrast to the previous study ${ }^{31}$ the numerical results here use purely forward-scattered data since the methods available to generate scattered data for the linear profile do not account for backscattering. However, the relative insensitivity of the method to small perturbations suggests that this is not crucial for grazing angles and moderate roughness where scattering angles and, therefore, backscattered energy are low. We have also found that decreasing the ratio $l / \Sigma$, i.e., reducing correlation length with respect to surface height, causes a loss of accuracy. This is partly due to the decrease in data resolution, but more significantly it eventually violates the assumptions underlying the parabolic equation, and the method breaks down. To a limited extent, this can be overcome by tuning the incident wavelength but highly diffuse multiple scattering remains a challenge. Increasing the strength of the linear profile also gradually degrades the performance, which we believe is due to the resulting increase in grazing angles which eventually challenges the parabolic equation assumption.

A more difficult question which merits further work is the extent to which the method copes with random variations in the refractive index in addition to the known deterministic component addressed here. An eventual goal is to develop this approach for three-dimensional problems and work is underway on this. The key elements of the algorithm extend naturally to three-dimensions, but the generation of scattered data to test the method requires further work.

${ }^{1}$ K. F. Warnick and W. C. Chew, "Numerical simulation methods for rough surface scattering," Waves Random Media 11(1), R1-R30 (2001). 
${ }^{2}$ A. G. Voronovich, Wave Scattering from Rough Surfaces (Springer Science \& Business Media, 2013), Vol. 17.

${ }^{3}$ M. Saillard and A. Sentenac, "Rigorous solutions for electromagnetic scattering from rough surfaces," Waves Random Media 11(3), 103-137 (2001).

${ }^{4}$ J. M. Elson, J. P. Rahn, and J. M. Bennett, "Relationship of the total integrated scattering from multilayer-coated optics to angle of incidence, polarization, correlation length, and roughness cross-correlation properties," Appl. Opt. 22(20), 3207-3219 (1983).

${ }^{5}$ D. L. Jordan and F. Moreno, "Enhanced backscattering and cross depolarization from multiscale surfaces," J. Opt. Soc. Am. A 10(9), 1989-1995 (1993).

${ }^{6} \mathrm{P}$. de Groot, "Principles of interference microscopy for the measurement of surface topography," Adv. Opt. Photonics 7(1), 1-65 (2015).

${ }^{7}$ S. Kuwamura and I. Yamaguchi, "Wavelength scanning profilometry for real-time surface shape measurement," Appl. Opt. 36(19), 4473-4482 (1997).

${ }^{8}$ R. J. Wombell and J. A. DeSanto, "The reconstruction of shallow roughsurface profiles from scattered field data," Inverse Problems 7(1), L7 (1991).

${ }^{9}$ R. J. Wombell and J. A. DeSanto, "Reconstruction of rough-surface profiles with the Kirchhoff approximation," J. Opt. Soc. Am. A 8(12), 1892-1897 (1991).

${ }^{10}$ I. Akduman, R. Kress, and A. Yapar, "Iterative reconstruction of dielectric rough surface profiles at fixed frequency," Inverse Problems 22(3), 939 (2006).

${ }^{11} \mathrm{~B}$. Zhang and H. Zhang, "Imaging of locally rough surfaces from intensity-only far-field or near-field data," Inverse Problems 33(5), 055001 (2017).

${ }^{12}$ Z. Cai, D. Chen, and S. Lu, "Reconstruction of a fractal rough surface," Phys. D: Nonlinear Phenom. 213(1), 25-30 (2006).

${ }^{13}$ Y.-Q. Jin and Z. Li, "Reconstruction of roughness profile of fractal surface from scattering measurement at grazing incidence," J. Appl. Phys. 89(3), 1922-1926 (2001).

${ }^{14} \mathrm{~J}$. Ya-Qiu, "Reconstruction of a heterogeneous fractal surface profile from scattering measurements at low grazing incidence," in 2005 IEEE Antennas and Propagation Society International Symposium (IEEE, 2005), Vol. 3, pp. 445-448.

${ }^{15} \mathrm{M}$. Spivack, "Solution of the inverse-scattering problem for grazing incidence upon a rough surface," J. Opt. Soc. Am. A 9(8), 1352-1355 (1992).

${ }^{16}$ M. Spivack, "Direct solution of the inverse problem for rough surface scattering at grazing incidence," J. Phys. A: Math. Gen. 25(11), 3295 (1992).

${ }^{17}$ C. D. Lines and S. N. Chandler-Wilde, "A time domain point source method for inverse scattering by rough surfaces," Computing 75(2-3), 157-180 (2005).

${ }^{18}$ M. Cayoren, I. Akduman, A. Yapar, and L. Crocco, "Shape reconstruction of perfectly conducting targets from single-frequency multiview data," IEEE Geosci. Remote Sens. Lett. 5(3), 383-386 (2008).
${ }^{19}$ J. T. Johnson, R. J. Burkholder, J. V. Toporkov, D. R. Lyzenga, and W. J. Plant, "A numerical study of the retrieval of sea surface height profiles from low grazing angle radar data," IEEE Trans. Geosci. Remote Sens. 47(6), 1641-1650 (2009).

${ }^{20}$ O. G. Nwogu and D. R. Lyzenga, "Surface-wavefield estimation from coherent marine radars," IEEE Geosci. Remote Sens. Lett. 7(4), 631-635 (2010).

${ }^{21}$ D. R. Lyzenga, O. G. Nwogu, R. F. Beck, A. O’Brien, J. Johnson, T. de Paolo, and E. Terrill, " Real-time estimation of ocean wave fields from marine radar data," in 2015 IEEE International Geoscience and Remote Sensing Symposium (IGARSS) (IEEE, 2015), pp. 3622-3625.

${ }^{22}$ D. Schiavulli, F. Nunziata, G. Pugliano, and M. Migliaccio, "Reconstruction of the normalized radar cross section field from GNSS-R Delay-Doppler Map,” IEEE J. Sel. Top. Appl. Earth Obs. Remote Sens. 7(5), 1573-1583 (2014).

${ }^{23}$ R. I. C. H. A. R. D. A. Paulus, "Evaporation duct effects on sea clutter," IEEE Trans. Antennas Propag. 38(11), 1765-1771 (1990).

${ }^{24}$ S. M. Babin, G. S. Young, and J. A. Carton, "A new model of the oceanic evaporation duct,” J. Appl. Meteorol. 36(3), 193-204 (1997).

${ }^{25} \mathrm{D}$. M. Milder, "Ray and wave invariants for sofar channel propagation," J. Acoust. Soc. Am. 46(5B), 1259-1263 (1969).

${ }^{26}$ F. B. Jensen, W. A. Kuperman, M. B. Porter, and H. Schmidt, Computational Ocean Acoustics (Springer Science \& Business Media, 2000).

${ }^{27} \mathrm{~J}$. A. DeSanto, "Exact boundary integral equations for scattering of scalar waves from infinite rough interfaces," Wave Motion 47(3), 139-145 (2010).

${ }^{28}$ F. D. Tappert, "The parabolic approximation method," in Wave Propagation and Underwater Acoustics (Springer, 1977), pp. 224-287.

${ }^{29}$ E. I. Thorsos, "Rough surface scattering using the parabolic wave equation,” J. Acoust. Soc. Am. 82(S1), S103-S103 (1987).

${ }^{30} \mathrm{M}$. Spivack, "A numerical approach to rough-surface scattering by the parabolic equation method," J. Acoust. Soc. Am. 87(5), 1999-2004 (1990).

${ }^{31}$ Y. Chen and M. Spivack, "Rough surface reconstruction at grazing angles by an iterated marching method," J. Opt. Soc. Am. A 35(4), 504-513 (2018).

${ }^{32}$ B. J. Uscinski, "Sound propagation with a linear sound-speed profile over a rough surface," J. Acoust. Soc. Am. 94(1), 491-498 (1993).

${ }^{33}$ M. Spivack and B. J. Uscinski, "Numerical solution of scattering from a hard surface in a medium with a linear profile," J. Acoust. Soc. Am. 93(1), 249-254 (1993).

${ }^{34}$ F. Tappert and L. Nghiem-Phu, "A new split-step fourier algorithm for solving the parabolic wave equation with rough surface scattering," J. Acoust. Soc. Am. 77(S1), S101-S101 (1985).

${ }^{35}$ J. D. Sheard, "Acoustic wave propagation in ice covered oceans," Ph.D. thesis (University of Cambridge, 1994).

${ }^{36}$ Y. Hatziioannou, "Scattering of an electromagnetic wave by a conducting surface," J. Mod. Opt. 46(1), 35-47 (1999).

${ }^{37}$ Y. Hatziioannou and M. Spivack, "Electromagnetic scattering by refractive index variations over a rough conducting surface," J. Mod. Opt. 48(7), 1151-1160 (2001) 